\title{
A Case of Drug - Induced Xerostomia and a Literature Review of the Management Options
}

Cuong Xuan Do* and Thang Nguyenphu

Barts and The London School of Medicine and Dentistry, New Road, London

*Corresponding author: Dr. Cuong Xuan Do, DDS, Post Graduate Student, Barts and The London School of Medicine and Dentistry, New Road, London, UK, Tel: 84944186999; E-mail: doxuancuong.87@gmail.com

Received date: June 07, 2017; Accepted date: July 20, 2017; Published date: July 27, 2017

Copyright: @ 2017 Do CX, et al. This is an open-access article distributed under the terms of the Creative Commons Attribution License, which permits unrestricted use, distribution, and reproduction in any medium, provided the original author and source are credited.

\begin{abstract}
A 56-year old man complained of oral dryness, impaired speech, eating and swallowing food. The past history and physical examination revealed that his current medication including tramadol, amitriptyline and simvastatin may cause his condition. Furthermore, a tendency to snoring and being stress possibly suggesting dry mouth would contribute the uncomfortable state. Investigations showed that the patient had true xerostomia without related systemic issues. Because of the complex reasons for the condition, a multidisciplinary approach was required in its prevention in order to achieve long-term stability.
\end{abstract}

Keywords: Drug-induced xerostomia; Dry mouth; Sjogren's síndrome; Guidelines

\section{Introduction}

The term "Xerostomia" is well known as a subjective complaint symptom of dry mouth and may be or may be not related to objectively measured hyposalivation. Several studies indicated that xerostomia is associated with a wide range of factors including local or systemic condition [1,2]; and thus, the degree of salivary dysfunction, as well as complication of dry mouth, may vary. These issues make xerostomia therapy complex and cost time to complete the treatment as well as to maintain stability after that. In addition, the condition is likely to be more severe amongst older patients as they could suffer from various diseases and take numerous involved medications that may result in a reduction of saliva secretion $[1,3]$. As a consequence, drug - induced xerostomia has still challenged clinicians regardless of how they could diminish appropriately the side - effects of medications that sometimes replacement should be considered.

\section{Case Report}

A 56-year old gentleman was referred to Royal London Dental Hospital on the 13th of June 2016 presenting complaint of dry mouth, impaired speech, eating and swallowing food.

Oral dryness was commenced approximately 12 months ago and was particularly noticed after speaking. His eating had not been particularly affected; however, he mentioned that he tended to need to drink to swallow food. He was not awoken at night with dryness and reported a tendency to snoring possibly suggesting mouth breathing. Also, he had not noticed any symptoms suggestive of eye dryness and had not noticed dryness of the skin as well as salivary gland swelling so far.

In addition to his major complaints, he had an upper partial removable denture for about three years, and recently felt difficulty in wearing it due to oral dryness. Also, several decay teeth on both jaws were applied amalgam filling in his last visit to his dentist about two months ago.

Regarding his past medical history, he had some osteoarthritis and had a right knee replacement from 2009. He continued to experience pain in the knees and shoulder. Past medical history of note includes hypercholesterolaemia, intestinal symptoms, occasional headaches, lowered mood, gout and osteoarthritis.

Current medication includes Tramadol, Allopurinol, Simvastatin, and Amitriptyline which he has been taking for three years.

Socially, he was a retired builder. He occasionally took alcohol and coffee, while he did not use tobacco. He had a mixed diet. Some stresses were found, and he is currently seeing a Counsellor.

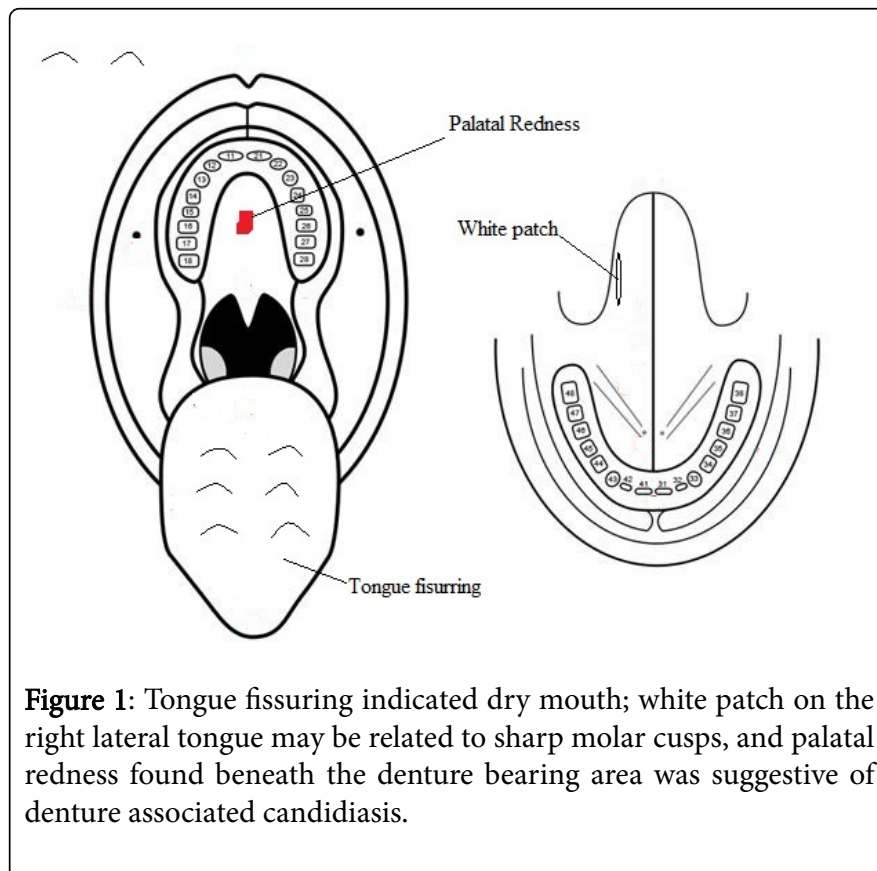


On examination, there was no salivary gland swelling or lymphadenopathy. Intra-orally, there was good plaque control. The oral mucous membranes appeared dry, and there was evidence of tongue fissuring and small homogeneous area of white change which may be related to trauma associated with sharp cusps of the lower right molar teeth. Also, he was partially dentate with an irretentive acrylic denture replacing his upper central and lateral incisors. Beneath his upper plastic denture as an area of redness which appeared confined to the denture bearing area (Figure 1).

Investigations revealed low unstimulated whole salivary flow rate of $0.08 \mathrm{ml} /$ minute (abnormally less than 0.1 ). Other blood tests including Full Blood Count (FBC), Erythrocyte Sedimentation Rate (ESR) and Immunology showed normal results. Similarly, the Glucose level in Urine, Schirmer's test (Eye test) and ultrasound of the salivary glands were not abnormal.

Xerostomia secondary to medications (common side effects of Tramadol, Amitriptyline and Simvastatin) was the diagnosis based on the data of mentioned history and investigations. False xerostomia caused by mouth breathing (snoring at night) was likely to contribute to the oral dryness, and it is possible that he has obstructive sleep apnoea.

A course of treatment was planned to manage patient's symptoms. He was provided advice to change his diet and habit: Frequent and regular sips of water; Avoidance of dry, hard, sticky and acidic foods; Leaving the acrylic denture out at night and giving denture hygiene instruction. Several salivary substitutes and lubricants (Saliva Orthana and BioXtra gel) were recommended. When it comes to other oral conditions, a replacement of poor fitting denture should be constructed. Sugar-free, Xylitol-containing mints, candies and gum may be advised to increase salivary flow.

Medically, Tramadol and Amitriptyline might be alternatives or reviewed the dose by his general practitioner (GP). Also, the patient was referred to discuss with his GP concerning obstructive sleep apnoea and stress.

After two weeks, the patient reported the slight improvement in his oral dryness achieved through the use of Biotene gel, Saliva Orthana spray and having frequent sips of water throughout the day. A new denture was made by a general dental practitioner (GDP). The palatal redness was alleviated significantly, and the white patch vanished; otherwise, the improvement of other managements was not recorded.

\section{Discussion}

The evidence-based review of patient's condition pointed out the major features of xerostomia and the managements of those symptoms.

A systematic review published in 2006 found that the prevalence of self - reported xerostomia in population - based samples ranged from $0.9 \%$ to $64.8 \%$ [4]. Although xerostomia can affect any age ranges, the onset is the most common amongst aged 65 years or older, accounting for $30 \%$ of this cohort [5].

Several causes came up in this condition could be categorised into false and true xerostomia. The term "false xerostomia" describes the sensation of dryness despite normal salivary output. In true xerostomia, the salivary flow is reduced. False xerostomia includes mouth breathing, mucosal disease, psychological disorders, whereas causes of true xerostomia consist of drugs, dehydration, Sjogren's syndrome, irradiation, neurological alterations, developmental anomaly [6].

Appropriate patient assessments including an inclusive medical and dental history, and a comprehensive head and neck examination both extra and intraoral - are essential for diagnosing xerostomia. The present patient with dry mouth described the need to sip fluids, especially when eating, difficulty in speaking and swallowing (deglutition). Such kinds of patients often have atrophic and erythematous oral mucosa, loss of papillae on the tongue and lips that peel and crack. Traumatic lesions may be visible on the buccal mucosa and the lateral borders of the tongue. Dentures may become loose, causing painful ulcerations. Regarding complication of xerostomia, dental caries and candidiasis can be observed in those patients. Enlargement of the major salivary glands, as well as salivary gland infection, may be revealed by inspection and palpation. Those signs and symptoms include swelling, discomfort, a cloudy or purulent discharge (pus), or a complete absence of clinically evident secretions [7].

In terms of medical problems, the present patient was suffering from multiple systemic diseases including osteoarthritis, hypercholesterolemia, intestinal symptoms, and gout. Although those diseases are unlikely to be causes of dry mouth, it is indicated that xerostomia could be a side effect of the medications used for medical issues. The incidence is high with Tramadol and Amitriptyline $[2,8]$ and most common oral manifestation when using Simvastatin [9].

Because there were many possible diagnoses for this case, the definite diagnosis was determined by carrying out several diagnosis tests. Unstimulated whole saliva showed a true xerostomia when it was less than $0.1 \mathrm{ml} / \mathrm{min}$. In some cases, the estimate of stimulated whole saliva below $0.7 \mathrm{ml} / \mathrm{min}$ is possibly used to be suggestive of salivary hypofunction. Blood test including FBC, ESR were also suggested when xerostomia was suspected to be related to systemic disease. Autoantibody screening and Schirmer's test were additional helpful investigations due to the fact that dry mouth may be associated with Sjogren's syndrome [7]. Furthermore, normal urine glucose level supported to exclude unsuspected diabetes as a cause of dehydration [6].

The treatment planning for xerostomia is often tailored by a multidisciplinary model of care including the following components: Patient education, management of systemic conditions and medication use, preventive measures, pharmacologic treatment with salivary stimulant and palliative measures. The prognosis may be positive as the outcome of treatment promoted his oral health after a course of 2week treatment. However, the management of this patient is still complicated and challenging to obtain the stability in long-term as the need for changing medication depends on his medical problems, and the decision will be made by his GP.

\section{Evidence-based review of treatment}

While some systemic reviews showed that the aetiology of xerostomia is numerous, and it is demanding to investigate the key cause for an individual case $[4,10]$, the dental staffs could follow American Dental Association guideline [7]. The latest guideline basing on strong evidence is to tailor dentists the methods to make diagnosis and treatment plan for the particular group of causes. 
Citation: Do CX, Nguyenphu T (2017) A Case of Drug - Induced Xerostomia and a Literature Review of the Management Options. Dentistry 7:

Page 3 of 3

\section{Conclusion}

Xerostomia has a variety of possible causes; and hence, the treatment for those requires sufficient co-operation between clinicians. For elderly patients, drug - induced xerostomia should be investigated thoroughly when taking their medical history as they often have various medical problems, and many medications are indicated to manage those issues. The treatment plan for those patients should be discussed with their doctors because it could affect their general health as well as other therapies.

\section{References}

1. McCreary C, Ni-Riordáin R (2010) Systemic diseases and the elderly. Dent Update 37: 604-607.

2. Stipetic MM (2012) Xerostomia - Diagnostics and treatment. Rad 514 Medical Sciences 38: 69-91.

3. Blain H, Rambourg P, Le Quellec A, Ayach L, Biboulet P, et al. (2015) Appropriate medication prescribing in older people. Rev Med Interne 36: 677-689.
4. Orellana MF (2006) Prevalence of xerostomia in population - based samples: a systematic review. J Public Health Dent 66: 152-158.

5. Ship JA, Pillemer SR, Baum BJ (2002) Xerostomia and the geriatric patient. J Am Geriatr Soc 50: 535-543.

6. Odell EW (2010) Dry mouth. Clinical Problem Solving in Dentistry, Churchill Livingstone, Elsevier Health Sciences, Elsevier Health Sciences UK, Edinburgh, pp: 33-36.

7. Plemons J, Al-Hashimi I, Marek C (2014) Managing xerostomia and salivary gland hypofunction Executive summary of a report from the American Dental Association Council on Scientific Affairs. J Am Dent Assoc 145: 867-873.

8. Looström H, Akerman S, Ericson D, Tobin G, Götrick B (2011) Tramadol-induced oral dryness and pilocarpine treatment: effects on total protein and IgA. Arch Oral Biol 56: 395-400.

9. Habbab KM, Moles DR, Porter SR (2010) Potential oral manifestations of cardiovascular drugs. Oral Diseases 16: 769-773.

10. Hanchanale S, Adkinson L, Daniel S, Fleming M, Oxberry SG (2015) Systematic literature review: xerostomia in advanced cancer patients. Support Care Cancer 23: 881-888. 